\title{
Some limitations on the use of distal cues in place navigation by rats
}

\author{
R. J. SUTHERLAND, G. L. CHEW, J. C. BAKER, and R. C. LINGGARD \\ The University of Lethbridge, Lethbridge, Alberta, Canada
}

\begin{abstract}
Place navigation by rats in the Morris (1981) water task was studied in two experiments. In Experiment 1, rats were trained to escape cool water by locating a goal platform in one half of a pool. Physical and/or visual access to the other half of the pool was restricted through the use of partitions. In subsequent transfer trials involving release from the restricted half, only those rats that had had prior unobstructed swimming and viewing experience in that region showed accurate transfer. In Experiment 2, viewing access to the room was allowed only during selected portions of the swim (beginning, middle, or after reaching the platform). Rats that were unable to view the room during the middle of the swim showed impaired acquisition. These results imply that rats acquire place information most efficiently while en route to the goal and that place navigation in a familiar environment from starting locations beyond familiar routes and views is inaccurate.
\end{abstract}

The neurobiological bases of spatial memories have received extensive attention recently from neuroscientists. Satisfactory progress in understanding the neural systems that underlie spatial learning and memory requires not only the collection of data about the relevant neuroanatomical and neurochemical machinery, but also a characterization of the nature of the information processing that these systems are specialized to accomplish. To take a simple example, the place-specificity of hippocampal single-unit firing in rats persists despite the absence of vision. The determination of whether rats can learn to navigate to a place in space from any direction in the absence of vision (Sutherland \& Dyck, 1984) restricts the plausible interpretations of this physiological fact.

In the present experiments we employed the place navigation task devised by Morris (1981). In this task rats must escape from cool water by swimming to a small invisible (i.e., submerged) platform in a large circular pool. The task has proven to be especially useful in assessing spatial learning and memory in rats with a variety of neurological manipulations. Several forms of hippocampal damage prevent normal performance in the task (Gage, Dunnett, Björklund, \& Stenevi, 1982; Morris, Garrud, Rawlins, \& O'Keefe, 1982; Sutherland, Kolb, \& Whishaw, 1982; Sutherland, Whishaw, \& Kolb, 1980, 1983). Damage to zones in the frontal neocortex, posterior neocortex, or neostriatum impairs acquisition of normal performance, albeit not in the same manner as does hippocampal damage (Dyck, Sutherland, \& Buday, 1985; Kolb, Sutherland, \& Whishaw, 1983; Sutherland \& Dyck, 1983;

This work was supported by an NSERCC grant to R. J. Sutherland. G. L. Chew is an NSERCC Postdoctoral Fellow. The authors thank R. H. Dyck, G. Cairns, B. E. Kolb, and G. T. Prusky for their assistance. Address correspondence to: R. J. Sutherland, Department of Psychology, The University of Lethbridge, Lethbridge, Alberta T1K 3M4, Canada.
Sutherland, Kolb, Becker, \& Whishaw, 1982; Sutherland, Kolb, \& Whishaw, 1982). In addition, the task has been employed in the study of the ontogeny of spatial mapping ability in young rats (Dyck et al., 1985; Rudy, StadlerMorris, \& Albert, 1987; Schenk, 1985) and its decline in aged animals (Gage, Kelly, \& Björklund, 1984). Certain pharmacological treatments (Morris, Anderson, \& Lynch, 1986; Sutherland, Whishaw, \& Regehr, 1982) and brain grafts (Gage et al., 1982) have also been shown to influence performance in this task. As the number of anatomical structures and neurochemical systems known to affect place navigation multiplies, it becomes increasingly important that we understand the nature of the competencies exhibited by rats when they learn and remember the location of a place in space. We assume that the manipulation of different neural components degrades (or enhances) the representation of different information, and that we will appreciate the significance of the aforementioned neurological experiments only if we can identify the nature of the information that is represented in each component system and how that representation is used in behavior.

We can distinguish three different strategies that rats use in moving from place to place: (1) a taxis strategy, which involves moving along a single, specific cue gradient, such as following an odor trail or approaching a visible landmark; (2) a praxis strategy, in which the rat performs a specific sequence of movements from some fixed starting location to reach a desirable end point; and (3) a mapping strategy, which, in its simplest manifestation, involves moving along a trajectory to a point defined by its relationship to at least two distal cues (O'Keefe \& Nadel, 1978; Sutherland \& Dyck, 1984). Rats may employ combinations of these strategies to solve a particular spatial problem. These three strategies differ in the kind of information necessary for their utilization, their rates of acquisition, their flexibility in the face of environ- 
mental changes, and almost certainly in their neurological representation.

It has been established that rats can solve the place navigation task readily using a mapping strategy, provided the invisible platform is in a fixed location relative to available distal cues (Morris, 1981; Sutherland \& Dyck, 1984). After reaching asymptotic performance, rats can swim directly to the invisible platform from any point on the pool's perimeter. It has been shown that rats can use both visual and auditory cues from the distal environment to aid navigation to the invisible platform, and that reducing the number of available distal cues impairs acquisition of accurate place navigation (Sutherland \& Dyck, 1984). During training, rats rear up and turn toward several directions when they climb onto the platform, and we have shown that rats can obtain important information about the location of the platform in certain circumstances at this time (Sutherland \& Dyck, 1984; Sutherland \& Linggard, 1982).

The present paper is concerned with establishing some of the conditions under which rats will acquire and use an effective mapping strategy to locate a hidden goal in the place navigation task.

In an experiment designed to demonstrate the robustness and flexibility of place navigation, Morris (1981) showed that if rats are trained to navigate to the invisible platform from only one starting point and then are transferred to a very different starting location on the pool's perimeter, they swim almost directly to the invisible platform from the first trial after transfer. The data suggest that "instantaneous transfer" (Morris, 1981, p. 258) of accurate navigation occurs from starting locations that were never used in training. Morris suggested that this phenomenon is one example of flexibility exhibited in spatial map learning by rats and that it indicates that spatial map learning may fundamentally differ from more traditional examples of instrumental or classical conditioning. However, if we reconsider Morris's experiment carefully we find that there are, in fact, several possible hypotheses concerning the mechansims that enable a rat to show this instantaneous transfer.

Consider the behavior of a well-trained rat released from only one starting location on the pool's perimeter. It swims quickly almost in a straight line to the invisible platform. The acceleration of its swim is virtually constant from trial to trial, relative to any available distal cue, and, in fact, on every trial it approaches a constant set of distal cues or a background cue located at the end point of a line that begins at the starting location and passes through the center of the invisible platform. In the most extreme case, if the animal at the time of transfer testing is released from the opposite side of the pool, accurate navigation requires that the direction of acceleration be reversed relative to every distal cue, and, in fact, the rat must swim away from the very background cue that it was approaching on previous trials. This phenomenon, as Morris (1981) noted, certainly presents a fundamental difficulty for simple stimulus-stimulus association accounts (Restle, 1957) or accounts that have been presented in the instrumental learning tradition (e.g., Skinner, 1969), but is entirely consistent with the cognitive mapping account of O'Keefe and Nadel (1978), in which 'the stored representation of the distal room cues permits the generation of novel directional behaviour' (Morris, 1981, p. 257) from a novel starting position.

So far we have omitted consideration of a potentially important aspect of the initial training of the rats in the Morris (1981) water task. On the first few trials of training, the rats certainly do not navigate directly to the platform. Rather, they swim more or less randomly into all sectors of the pool, with an initial bias toward the wall of the pool. Rarely are they consistent in approaching the invisible platform from the direction of the initial starting location. Thus, by the time a rat has acquired accurate place navigation it has viewed the room from nearly all possible vantage points in the pool, as well as having approached the platform from numerous directions. The question arises, then, What is the contribution of incidental viewing from, or incidental passage through, a region during initial learning to later navigation specifically involving that region?

The answer to this question has important implications for the understanding of the rat's spatial mapping system. If a rat can generate novel, appropriate directional behavior from an area from which it has never viewed the available distal cues, or through which it has never navigated, then the rat's mapping system must be of the type outlined by O'Keefe and Nadel (1978). That is, on the basis of a restricted set of views of the environment, as well as a restricted set of trajectories through that environment, rats generate a topographical representation of the available distal cues and platform position. This map, in turn, can be used in the calculation of accurate novel trajectories to the goal from all possible locations in the environment.

Unfortunately, reconsideration of Morris's (1981) experiment does not provide convincing evidence that rats do have this ability. In particular, it may be the case that rats cannot generate accurate trajectories from a novel area without (1) having viewed the distal cues from that area, or (2) having traveled through that area as part of previous trajectories to the goal.

If either of these conditions is necessary to produce successful navigation, then the notion of generation of trajectories based upon extrapolations from information contained within some unitary topographical map is less compelling. A more plausible explanation would be that the generation of a relatively novel trajectory is based upon an interpolation within a familiar range of views of distal cues and trajectory outcomes that the rat has actually experienced. Such a notion is similar to those found in the human cognition literature (Brooks, 1978; Medin \& Schaffer, 1978).

\section{EXPERIMENT 1}

In Experiment 1, we systematically varied the experience rats had in viewing distal cues from, as well as 
swimming through, a selected region of the pool. In so doing we attempted to establish whether accurate navigation to the invisible goal from a particular region requires either (1) prior experience in viewing distal cues from that region or (2) travel through that region as part of a swim trajectory to the invisible platform.

\section{Method}

\section{Subjects}

Twenty-eight male hooded rats of the Long-Evans strain, weighing $300-400 \mathrm{~g}$, served in this study. They were housed in pairs, with continuous access to Purina rat chow and water, under a 12-12 lightdark cycle. All behavioral testing was conducted during the light phase of the cycle.

\section{Apparatus}

The water maze pool was circular ( $1.5 \mathrm{~m}$ in diameter), with a white interior. A $13 \times 13 \mathrm{~cm}$ clear Plexiglas platform was always positioned in the center of the southeast quadrant of the pool. The top surface of the platform was $2 \mathrm{~cm}$ below the surface of the water. The water in the pool was made cloudy by dissolving 1.5 liter of instant skim milk power in it. For some experimental conditions, a clear Plexiglas partition ( $1.6 \mathrm{~m}$ wide $\times 0.75 \mathrm{~m}$ high) was placed across the east-west diameter of the pool, and for other experimental conditions, an opaque black curtain was hung from floor to ceiling, wall to wall, across the east-west diameter of the pool.

Measurements of navigation on every trial were recorded using an automated target tracking system. Briefly, the rats were observed by means of a black-and-white video camera positioned above the center of the pool. The output of the camera was provided to an HVS Image Analysis unit (Model VP112), which calculated the $x-y$ coordinates of the position of the rat's head. The coordinates were sent to an Apple II+ computer at the rate of $50 / \mathrm{sec}$ and were stored on twinned Xebec 10-MB hard disk drives.

\section{Procedure}

The latency to reach the platform, the length of the swim path, and the initial heading error, measured at the point where the rat had completed the first $30 \mathrm{~cm}$ of the swim path, were recorded for every trial. The latter measure was calculated by drawing an imaginary line from the starting location on a particular trial through the animal's position after it had swum $30 \mathrm{~cm}$ and measuring the angle subtended by this line and a second line drawn from the starting location to the center of the platform.

The rats were randomly assigned to seven groups $(n=4)$. The groups differed in the experience provided them in the north half of the pool (Region B, Figure 1).

Training. Group A rats were trained to swim from two starting locations at the perimeter of the pool: one in the southwest, the other in the southeast (see Figure 1). The clear partition bisected the pool in the east-west direction. Its bottom edge was positioned $7 \mathrm{~cm}$ above the surface of the water. The training phase consisted of 24 trials. Eight trials were conducted daily; within each block of 4 trials the rats were released from each of the two starting locations twice in a pseudorandom sequence. Group B rats were treated identically except that the two starting locations were at the pool's perimeter in the northwest and northeast. The rats of Group $C$ were treated the same as those of Group A except that the bottom edge of the clear partition was lowered into the water to prevent the rats from swimming into Region B. The rats of Group D were treated the same as those of Group $C$ except that, following the end of the training trials each day, they were placed into Region B with access to Region A blocked by the clear partition.

The length of time Group D rats spent in Region B was yoked to comparable performance by the Group $A$ rats. Thus, if the first rat of Group A swam for a total of $30 \mathrm{sec}$ on trial 1 of training, of which $15 \mathrm{sec}$ were spent in Region $B$, then the first rat of Group D, after its training trials, was placed into Region $B$ and allowed to swim for $15 \mathrm{sec}$. This would be repeated for the second rats of Groups D and A, and so on, for all eight of Group A's training trials. The rats of Group $E$ were treated the same as those of Group D except that when they were placed in Region B the opaque curtain was placed across the east-west diameter of the pool. Group F rats were treated the same as those of Group A except that the opaque curtain was placed across the east-west diameter of the pool. The rats of Group $G$ were treated the same as those of Group $C$ except that the opaque curtain was placed across the east-west diameter of the pool.

Transfer test day. All rats were treated identically during the block of four transfer trials on Day 4 of testing. The rats were released twice from the northwest and twice from the northeast starting locations in a pseudorandom sequence, without the clear partition or the opaque curtain. All rats were treated identically during the block of four trials of posttransfer testing. They were released twice from the southwest and twice from the southeast starting locations.

\section{RESULTS}

Figure 2 depicts the mean latency to find the invisible platform for each block of trials during pretransfer training, the transfer trial block, and the posttransfer trial block. Figure 3 presents one representative swim path from each experimental group on the last block of trials before transfer and on the transfer trial block.

A multivariate analysis of variance (MANOVA) of latency and initial heading error data was performed using the BMDP81-4V statistical package on the University of Lethbridge DEC-20 computer system. The design of the analysis involved one between-groups factor (the seven experimental groups) and one within-groups factor (the first six trial blocks). No significant differences in either mean latency or initial heading error were found between groups in any of the six trial blocks preceding the transfer test day. That is, the asymptotic performance and rate of acquisition were similar in all groups, despite the differences in training environment among the groups.

The asymptotic latency and heading error of each group in the sixth trial block were taken as the most representative measures of accuracy of navigation during the baseline period. These pretransfer day measures were used in a second analysis along with the corresponding measures made during the transfer and posttransfer trial blocks. The design of this MANOVA involved one between-groups factor (the seven experimental groups) and one within-groups factor (pretransfer, transfer, and posttransfer trial blocks).

A significant overall main effect between groups was found [Wilks lambda $=0.38$, corresponding to an approximate $F(12,40)=2.06, p<.05]$. The subsequent univariate test on latency was also significant $[F(6,21)=$ $2.66, p<.05$ ]. The univariate test on heading error, on the other hand, was not significant $(F<1)$.

A significant overall within-groups effect was also found [Wilks lambda $=0.68$, corresponding to an approximate $F(4,82)=5.1, p<.05]$. The subsequent univariate test on latency was significant [Greenhouse-Geisser adjusted 


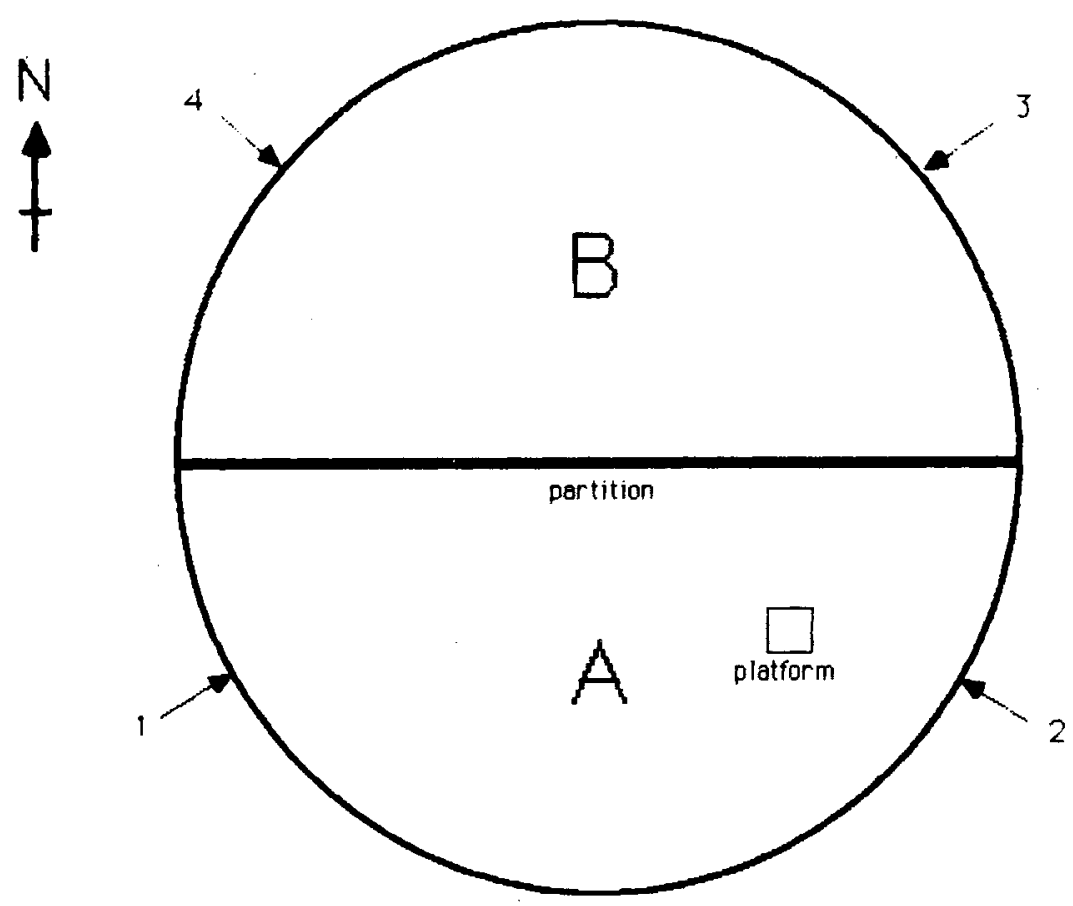

GROUP

$A$ - Start at $1 \& 2$, access to $A \& B$, clear partition.

$B$ - Start at $3 \& 4$, access to $A \& B$, clear partition.

C - Start at $1 \& 2$, access to A only, clear partition.

D - Start at $1 \& 2$, access to A only, forced swim in B, clear partition.

$E$ - Start at 1 \& 2, access to A only with clear partition, forced swim in B with opaque partition.

$F$ - Start at $1 \& 2$, access to $A \& B$, opaque partition.

$G$ - Start at $1 \& 2$, access to $A$ only, opaque partition.

Figure 1. The arrangement of partitions, platform, and starting locations with a description of the conditions for each group in Experiment 1.

$F(1.3,27.6)=7.83, p<.01]$. The univariate test on within-group differences with respect to heading error was also significant [Greenhouse-Geisser adjusted $F(2,0,41.7)$ $=5.22, p<.01]$. Further univariate analysis revealed that the source of variation was attributable to differences in latency $[F(1,21)=17.8, p<.0004]$ and heading error $[F(1,21)=10.5, p<.004]$ between the pretransfer and transfer trial blocks. In contrast, latency and heading error differences between pre- and posttransfer trial blocks were not statistically significant.

To more fully analyze differences among the groups on the transfer trials, we carried out post hoc comparisons, using Fisher's $L S D$ method with $p=.05$ in each case. With respect to latency, we found that all groups were equal on the pretransfer trial block. On the transfer trial block, Group A and Group B rats (which during training could swim through the entire pool with an unobstructed view of the distal cues) did not significantly differ from each other, but all of the remaining groups had significantly longer latencies than did Groups $A$ and B. Similarly, with respect to heading error, there were no group differences on the pretransfer trial block. The rats of Group B (which were released from the transfer starting locations during training) were the only rats that maintained a low value for heading error from the pretransfer to transfer trial blocks $\left(13.0^{\circ}\right.$ vs. $\left.13.8^{\circ}\right)$. All of the remaining groups had statistically significantly higher heading errors than did Group B on the transfer trial block (the values for these groups ranged between $30^{\circ}$ and $39^{\circ}$ ).

Transfer trial block performance of Groups D and E was compared with that of Groups $C$ and $G$ (the former 

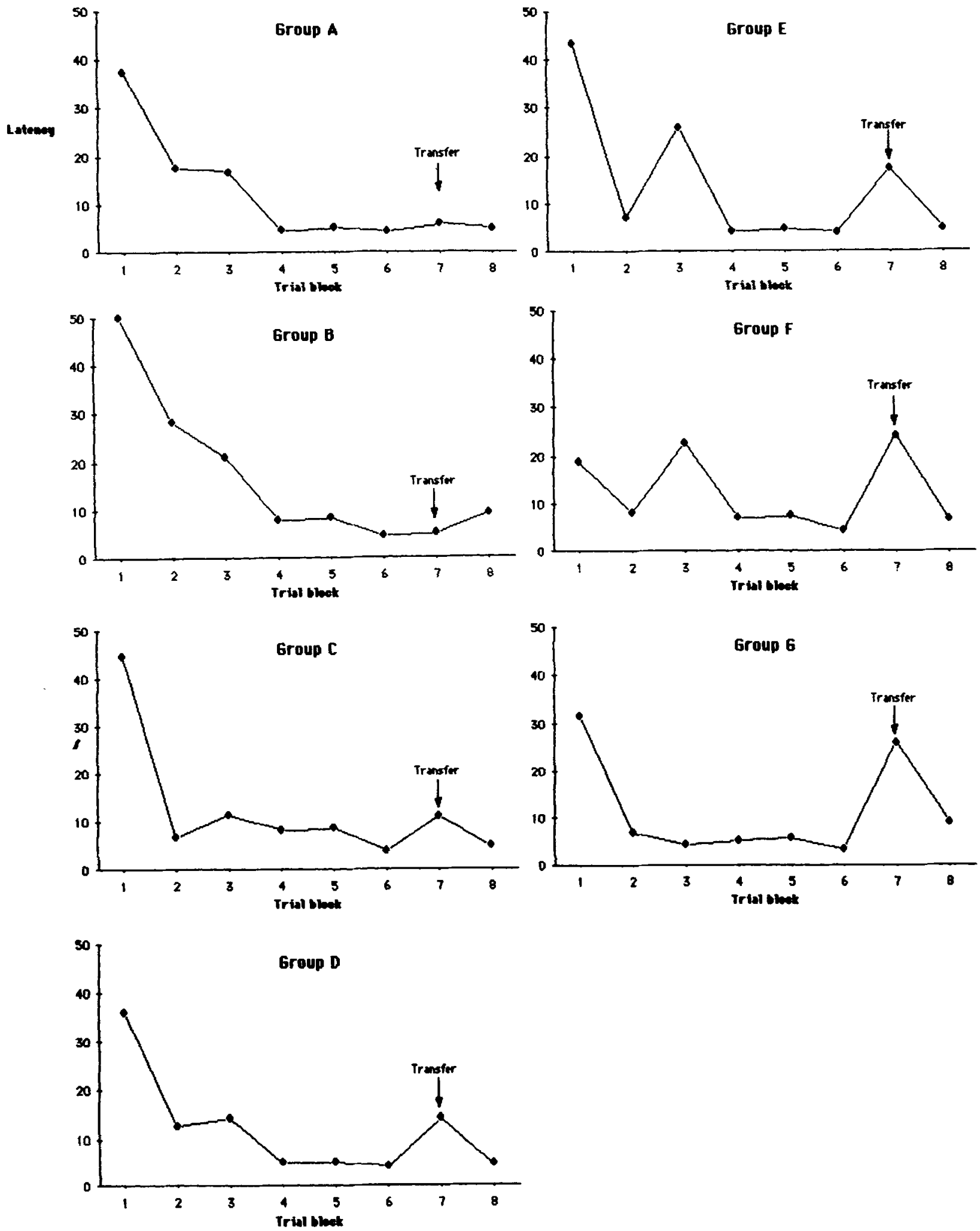

Figure 2. The mean latency to find the platform on each block of four trials for each group in Experiment 1. 


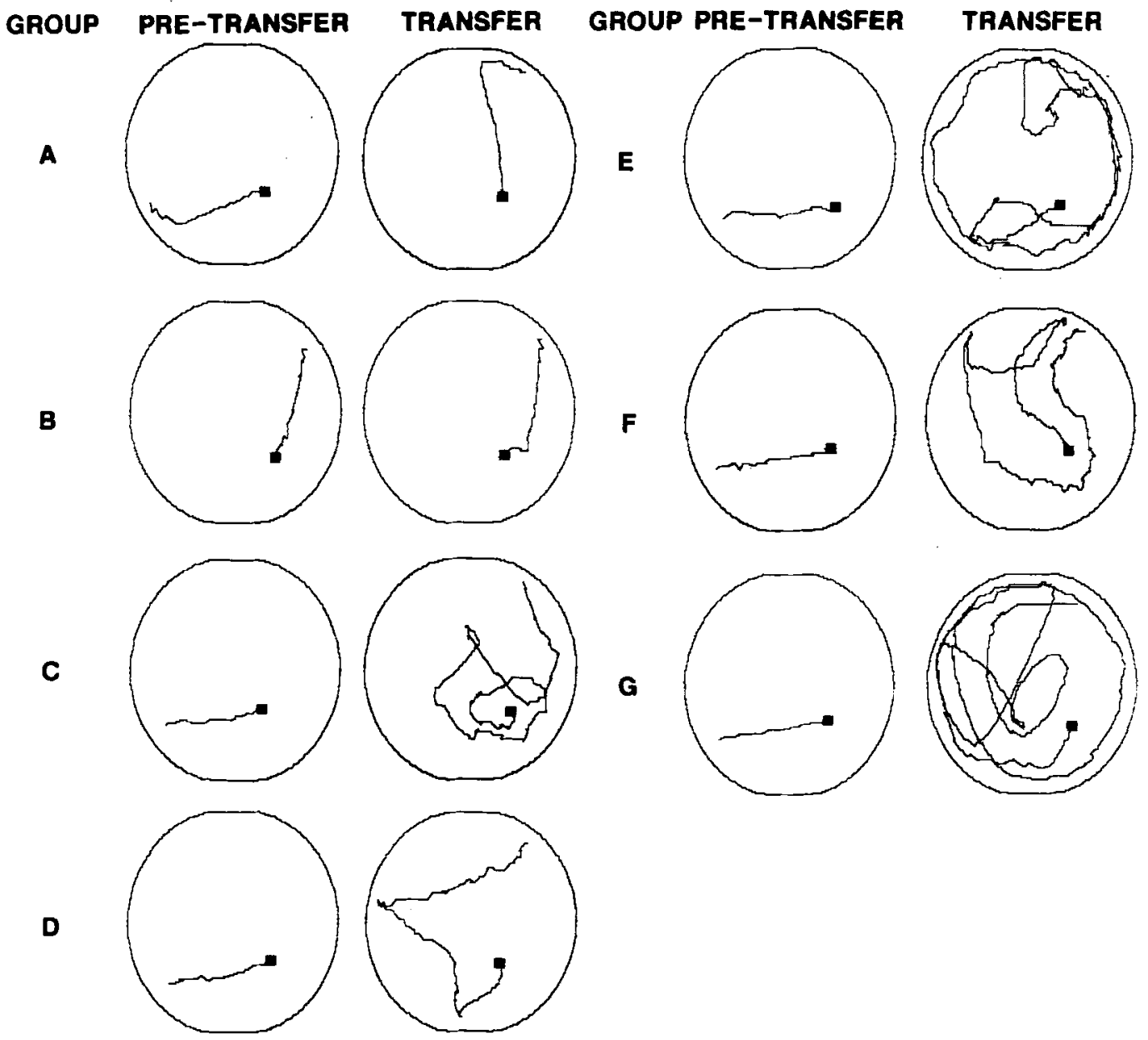

Figure 3. A swim path from each group in Experiment 1, representing median performance from the block of four trials before transfer testing and from the block of transfer trials.

two groups were treated identically to the latter two groups except that they received forced swims in Region B of the pool). There were no statistically significant differences in latency and heading error. Post hoc comparisons between the groups that had been trained with the opaque partition (Groups E, F, and G) and the groups that were treated similarly but had been trained with the clear partition (Groups B, C, and D) revealed that the former groups had statistically significantly longer latencies than did the latter groups on the transfer trial block. Similar comparisons for heading error were not statistically significant.

In order to rule out the possibility that the observed differences in latency among the groups were produced by variations in swimming speed on the transfer test, we calculated speed of swimming during the trials of the transfer test. This calculation revealed that all groups had similar swimming speeds, and, in fact, that the two groups with the shortest latencies exhibited the slowest swimming speeds ( $9 \%$ slower than the mean swimming speed of all rats during transfer trials). The two groups with the longest latencies exhibited swimming speeds that were $5 \%$ faster than the overall mean speed. Thus, differences in swimming speed cannot account for the observed differences in latency.

Inspection of the transfer day latencies (Figure 2) confirms these clear group differences. These differences indicate that only the rats of Groups A and B, which had had both unrestricted swimming access to and unrestricted viewing of the entire pool, showed relatively efficient trajectories during the transfer trials (Figure 3).

In summary, the rats in all experimental conditions acquired place navigation to the invisible platform at similar rates to similar asymptotic levels of performance. Despite the absence of significant differences in performance during training, the groups did differ in performance on the transfer trials. Specifically, the rats of groups that either had not viewed the room from the region of the transfer starting locations or had not had the opportunity to swim through this region as part of a swim path ending at the platform showed longer latencies to reach the platform and exhibited less accurate initial headings on the transfer trials. In addition, even the rats that had been able to swim through the entire pool in full view 
of the distal cues showed significantly less accurate initial headings when they were released from the novel transfer starting locations. Providing rats with a forced swim during training in the half of the pool containing the novel transfer starting locations had no reliable effect, but blocking viewing access to all of the distal cues with the opaque partition produced significantly worse transfer performance, as measured by latency to find the platform.

\section{EXPERIMENT 2}

The results of Experiment 1 imply that prior viewing of distal cues while navigating through a particular region to a goal site is necessary for accurate navigation from that region to the goal. In Experiment 2 we examined this phenomenon further by attempting to identify the portion of a swim trial during which distal cue information is obtained. We darkened the entire test area for selected segments of the swim, but permitted unrestricted access to the entire pool. On the basis of our prior observation of many rats during acquisition of place navigation, we divided the swim into three segments: (1) an initial 1-sec segment immediately following entry of the rat into the pool, during which time rats nearly always initiate swimming in a certain direction; (2) a middle segment, ending with the rat's placing its forepaws onto the submerged platform; and (3) a final segment, lasting $15 \mathrm{sec}$, after which the rat was removed from the platform. If the implications of Experiment 1 have generality, then the acquisition of place navigation using visual cues should be predominantly affected by the illumination of distal cues during the middle segment of the swim. Ilumination of distal cues at the beginning or end of the swim should have little or no effect on acquisition of place navigation.

Method
Subjects
Thirty-two male Long-Evans hooded rats, housed and maintained
as in Experiment 1, were used.

\section{Apparatus}

The pool described in Experiment 1 was used. During the darkened conditions the pool was weakly illuminated by a $15-\mathrm{W}$ red light bulb, coated with black paint, suspended $2 \mathrm{~m}$ above the center of the pool. The ceiling and walls of the room were not illuminated by the red light, but the illumination was just bright enough to allow a dark-adapted experimenter, who was seated near the edge of the pool, to trace the rats' swim path onto a diagram of the pool. During the lighted conditions, the entire test area was brightly illuminated by fluorescent fixtures on the ceiling, controlled by an experimenter via a foot switch. Two constant white noise sources were positioned on opposite sides of the pool and a metronome that emitted a $2-\mathrm{Hz}$ click was placed in one corner of the room. These noise sources and a cart on which the rats remained between trials were positioned in the same places for all but the final day of testing.

\section{Procedure}

The rats were randomly assigned to eight groups of 4 animals each. Each group corresponded to a particular combination of lightson/lights-off during the initial, middle, and final segments of a place navigation trial. For one group, all segments of the swim were illuminated, whereas for another group, only the initial and middle segments of the swim were illuminated, and so on.

An experimenter sat at the edge of the area illuminated by the red light and recorded each rat's swim path and latency to find the platform. A second experimenter placed the rats, one at a time, into the pool, facing the wall, at the four cardinal compass points. During four daily trials the rats were released from each starting location once in a random sequence. The maximum trial duration was $90 \mathrm{sec}$. When a rat located the submerged platform, it was allowed to remain on the platform for $15 \mathrm{sec}$. The rats were prevented from dark adapting by illuminating the room between trials. During this time the experimenter who recorded the data covered his eyes. The initial segment of thte swim was timed using the metronome. The rat was placed into the pool on a beat and the initial segment ended two beats later.

The rats were tested for 9 days. For the first 8 days all conditions were constant, but for the four trials on the ninth day, the positions of the cart, the two white noise sources, and the metronome were rotated $90^{\circ}$ relative to the fixed positions of the pool and platform. This manipulation was included in order to assess the relative reliance upon auditory and nonauditory information by rats in the different treatment groups.

\section{Results}

The latency data were analyzed by a repeated measures analysis of variance procedure (all $p s=.001$ ) using the BMDP79-8V statistical package on the University of Lethbridge DEC-20 computer. The data were in a $2 \times 2 \times 2$ design corresponding to the lighting condition (lights on or off) in each of the three swim segments. On the first day there were no significant differences among the groups. All $F$ ratios fell below significance, with tail probabilities greater than 0.1 . However, a comparison among groups on Day 8 revealed a significant main effect of lighting during the middle segment of the $\operatorname{swim}[F(1,24)$ $=19.9 \mathrm{]}$. Middle segment lighting did not significantly interact with any other factor. A comparison of performance of all groups on Day 8 with that observed on Day 9 revealed a significant main effect of middle segment lighting $[F(1,24)=19.10]$, a significant effect of day $[F(1,24)$ $=18.6]$, and a significant interaction between middle segment lighting and day $[F(1,24)=7.65, p<.01]$.

Figure 4 shows the average latency on each day for the two levels of lighting for each of the three segments of the swim. During training, the groups for which the room was illuminated during the middle segment had shorter latencies than the other groups. Lighting conditions during the other segments had no effect, nor did they interact significantly with middle segment lighting. The significant interaction of middle segment illumination with day was due to the fact that all groups without room illumination during the middle segment were significantly slower in finding the platform when the sound sources were moved, but those groups that could see the room during the middle segment were unaffected.

Thus, the opportunity to view the room during the middle segment of the swim was the only factor to significantly affect the acquisition of place navigation. This is demonstrated by shorter latencies to find the submerged platform and by the greater resistance in probe trials to 

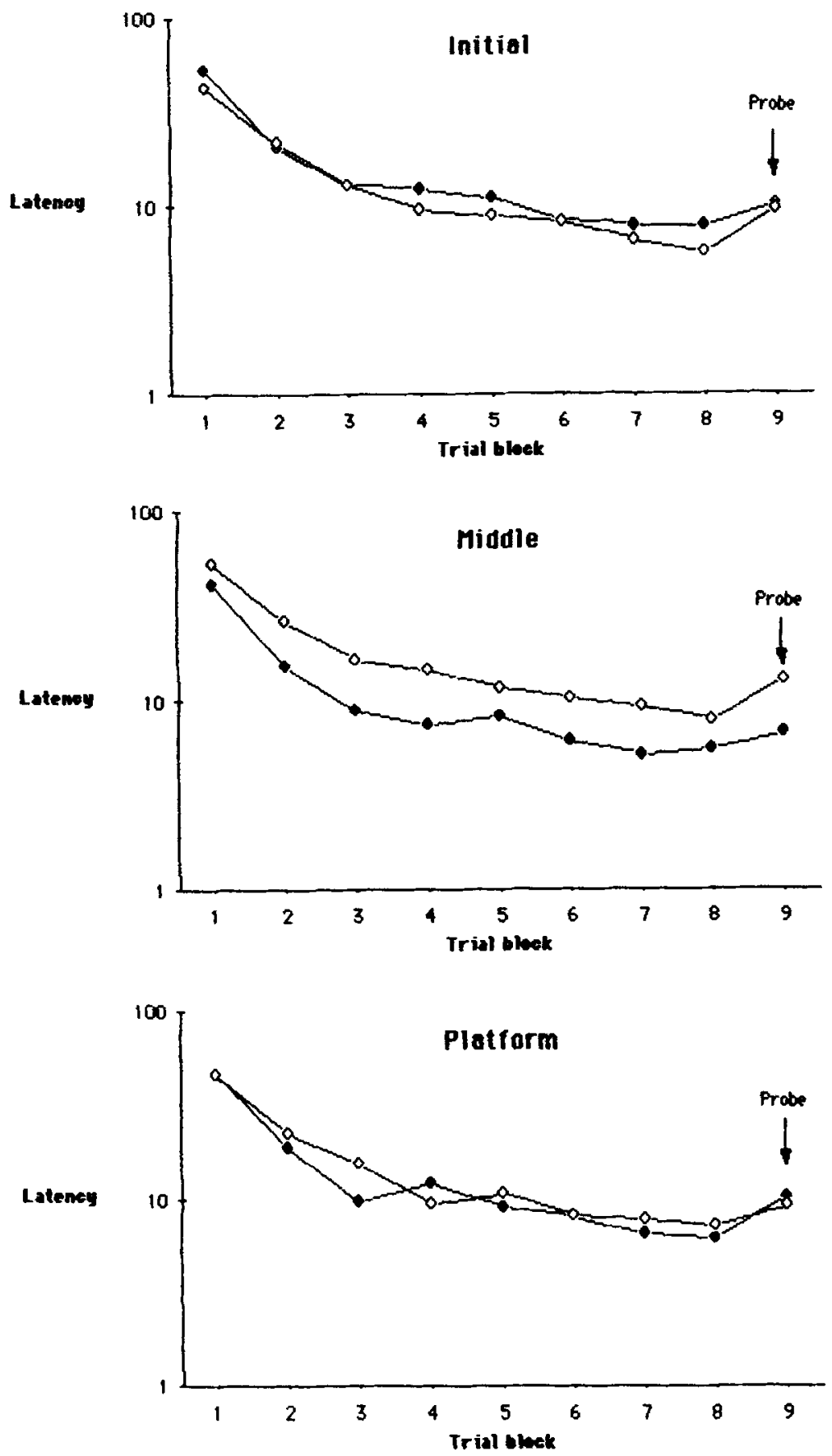

Figure 4. The mean latency to find the platform for each level of lighting in the three swim segments in Experiment 2 (filled circles indicate lights on, open circles indicate lights off). The probe test was conducted on the ninth block of trials when the sound sources were rotated relative to the room. 
Table 1

Mean Latency (in Seconds) to Find the Hidden Platform for Each Group

\begin{tabular}{cccc}
\hline & \multicolumn{3}{c}{ Trial Block } \\
\cline { 2 - 4 } Group & 1 & 8 & 9 \\
\hline 000 & 48.5 & 6.9 & 10.6 \\
100 & 58.0 & 8.8 & 13.1 \\
010 & 39.1 & 5.6 & 7.5 \\
001 & 41.8 & 4.4 & 12.8 \\
011 & 41.4 & 5.4 & 7.4 \\
101 & 60.6 & 7.6 & 14.9 \\
110 & 40.8 & 4.8 & 6.1 \\
111 & 44.1 & 5.2 & 5.8 \\
\hline
\end{tabular}

Note-Three-digit group codes indicate room darkened (0) or lit (1) for each of the three phases of the swim.

disruption caused by discordant auditory information exhibited by the groups that were permitted to view the room during the middle swim segment.

\section{DISCUSSION}

The results we obtained in Experiment 1 with rats trained to navigate to the invisible platform from starting locations on only one side of the pool, but which had unrestricted viewing of the room and unrestricted swimming access to the entire pool, were consistent with the results obtained by Morris (1981) under similar conditions. In transfer tests, when these rats were required to navigate from novel starting locations on the opposite side of the pool, they took no longer to swim to the invisible platform than the rats that had been started at those locations consistently throughout training. Under these conditions, at least, we were able to demonstrate good transfer of place navigation from novel starting locations and novel directional behavior. However, we did find that the accuracy of the direction of the initial segment of the swim was significantly worse for these rats than for rats that had been released from the transfer starting locations during training. Thus we were unable to demonstrate complete and instantaneous transfer of accurate directionality to the novel starting locations.

Rats that were prevented from swimming in both halves of the pool in a continuous trajectory, as well as those that were prevented from simultaneously viewing both halves of the room, were unable to navigate efficiently to the invisible platform from the novel starting locations during transfer tests. We have evidence that the minimal obstruction of viewing associated with the presence of the clear partition across the pool did not by itself disrupt performance. The rats that could swim under it during training (Groups A and B) demonstrated efficient performance on the transfer trials. On the other hand, the rats that were prevented from swimming in half of the pool by the clear partition (Group C) failed to show accurate navigation from the transfer starting sites. The rats of this group could readily view all of the distal cues in the room, but were denied the opportunity to view them from half of the pool and to swim in a continuous trajectory through both halves of the pool. We can conclude that both of these factors contributed to their poor transfer performance, inasmuch as we found poor transfer performance by the rats that could swim in a continuous trajectory through both halves of the pool, but could not view the entire room from both sides of the pool (Group F). Poor transfer performance was also shown by the rats that could view the entire room from both sides of the pool, but could not swim in a continuous trajectory through both halves of the pool (Group D). Although the rats of Group C showed disrupted performance during transfer testing, it is probably the case that they exhibited some "savings," because their navigation from the novel starting locations was superior to that of some of the other groups and was better than their performance on the first trials of acquisition. It may not be possible to reliably assess the nature and degree of any possible savings in this group, because it is not obvious which is the appropriate comparison condition for such an assessment. It is important to note that the rats that during training experienced a forced swim in the vicinity of the transfer sites might have learned that they could not escape from the water on that side of the pool. That this did not actually occur is suggested by the finding that these rats were no worse on transfer trials than the rats that were treated identically but did not experience forced swims.

Thus, in order to exhibit transfer of efficient place navigation from a novel starting location in this situation, it is necessary during training for the rats to (1) be familiar with the distal cues viewed from the region of the novel starting location, and (2) swim through the vicinity of the novel starting location as part of a swim path associated with the invisible goal. It would require a more extensive experimental design to reliably characterize the relative contributions of these two factors to transfer performance. However, in the present results the impairment produced by permitting only restricted views of distal cues is striking.

Further support for our general conclusion was obtained in Experiment 2. The only main effect of the experimental manipulations was the improvement in acquisition of place navigation afforded by viewing the room during the middle segment of the swim. The opportunity to view the room during the initial segment of the swim and/or from the platform did not significantly affect acquisition of place navigation. Similarly, only illumination of the room during the middle of the swim protected place navigation from disruption by discordant auditory information. It is important to note that, at least in some circumstances, rats can benefit from information obtained by viewing the room from the submerged platform (Sutherland \& Linggard, 1982). In the cases in which such a benefit was detected, the rats had previously navigated around the entire pool with unrestricted viewing access to the entire room. Given our results, this previous experience must be a necessary condition for the rats' use of the information obtained while standing on the platform.

Our findings point to important limitations on the flexibility of the use of distal visual information in spatial mapping when rats learn to navigate from place to place in 
an unfamiliar environment. One implication of our results is that rats may be able to readily generate novel and appropriate trajectories only from novel starting locations that are within the range of routes and views of the available distal cues that the rats have actually experienced.

\section{REFERENCES}

Brooks, L. R. (1978). Non-analytic concept formation and memory for instances. In A. D. Baddeley \& J. Long (Eds.), Attention and performance IX. Hillsdale, NJ: Erlbaum.

Dyck, R. H., Sutherland, R. J., \& Buday, M. R. (1985). The ontogeny of mapping and non-mapping spatial strategies following neonatal hippocampal damage in rats. Society for Neuroscience Abstracts, 11,832 .

GaGe, F. H., Dunnett, S. B., BuöRklund, A., \& Stenevi, U. (1982). Functional changes following embryonic grafts to the deafferented hippocampal formation. Society for Neuroscience Abstracts, 8, 1979.

GaGe, F. H., Kelly, P. A. T., \& Buörklund, A. (1984). Regional changes in brain glucose metabolism reflect cognitive impairments in aged rats. Journal of Neuroscience, 11, 2856-2865.

Kolb, B., Sutherland, R. J., \& Whishaw, I. Q. (1983). A comparison of the contributions of the frontal and parietal cortex to spatial localization in rats. Behavioral Neuroscience, 97, 13-27.

Medin, D. L., \& SCHAFFer, M. M. (1978). Context theory of classification learning. Psychological Review, 85, 207-238.

Morris, R. G. M. (1981). Spatial localization does not require the presence of local cues. Learning \& Motivation, 12, 239-260.

MorRIs, R. G. M., ANDERSON, E., \&YNCH, G. S. (1986). Selective impairment of learning and blockade of long-term potentiation by an $N$-methyl-D-aspartate receptor antagonist. Nature, 319, 774-776.

Morris, R. G. M., Garrud, P., Rawlins, J. N. P., \& O'Keefe, J. (1982). Place navigation impaired in rats with hippocampal lesions. Nature, 297, 681-683.

O'KeEFE, J., \& NADEL, L. (1978). The hippocampus as a cognitive map. Oxford: Clarendon Press.

RESTLE, F. (1957). Discrimination of cues in mazes: A resolution of the "place vs response" question. Psychological Review, 64, 217-228.
Rudy, J. W., Stadler-Morris, S., \& Albert, P. (1987). Ontogeny of spatial navigation behaviors in the rat: Dissociation of "proximal" and "distal"-cue-based behaviors. Behavioral Neuroscience, 101, 62-73.

SCHENK, F. (1985). Development of place navigation in rats from weaning to puberty. Behavioral \& Neural Biology, 43, 69-85.

SKINNER, B. F. (1969). Contingencies of reinforcement. New York: Appleton-Century-Crofts.

SutHerland, R. J., \& DYCK, R. H. (1983). Hippocampal and neocortical contributions to spatial learning. Society for Neuroscience $A b$ stracts, 9, 638.

SUTherland, R. J., \& Dyck, R. H. (1984). Place navigation by rats in a swimming pool. Canadian Journal of Psychology, 38, 322-347.

Sutherland, R. J., Kolb, B., Becker, J. B., \& Whishaw, I. Q. (1982). Cortical noradrenaline depletion eliminates sparing of spatial learning after neonatal frontal cortex damage in the rat. Neuroscience Letters, 32, 125-130.

Sutherland, R. J., KolB, B., Whishaw, I. Q. (1982) . Spatial mapping: Definitive disruption by hippocampal or medial frontal cortical damage in the rat. Neuroscience Letters, 31, 271-276.

Sutherland, R. J., \& LingGard, R. C. (1982). Being there: A novel demonstration of latent spatial learning in the rat. Behavioral \& $\mathrm{Neu}$ ral Biology, 32, 103-107.

SUTHERLAND, R. J., Whishaw, I. Q., \& KolB, B. (1980). Abnormalities in EEG and spatial performance following intrahippocampal injections of neurotoxins. Society for Neuroscience Abstracts, 6, 565.

Sutherland, R. J., Whishaw, I. Q., \& Kolb, B. (1983). A behavioural analysis of spatial localization following electrolytic, kainate-, or colchicine-induced damage to the hippocampal formation in the rat. Behavioural Brain Research, 7, 133-153.

Sutherland, R. J., Whishaw, I. Q., * Regehr, J. C. (1982). Cholinergic receptor blockade impairs spatial localization using distal cues in the rat. Joumal of Comparative \& Physiological Psychology, 96, 563-573.

(Manuscript received July 17, 1986; revision accepted for publication January 27,1987 .) 